\title{
Association of monocyte HLA-DR expression over time with secondary infection in critically ill children: a prospective observational study
}

\author{
Nienke N. Hagedoorn ${ }^{1}$ - Pinar Kolukirik ${ }^{2}$. Nicole M. A. Nagtzaam ${ }^{3}$ - Daan Nieboer ${ }^{4} \cdot$ Sascha Verbruggen $^{5}$. \\ Koen F. Joosten ${ }^{5} \cdot$ Henriette Moll ${ }^{1} \cdot$ Gertjan Driessen $^{6} \cdot$ Willem A. Dik $^{3} \cdot$ Clementien Vermont $^{2}$
}

Received: 16 June 2021 / Revised: 26 October 2021 / Accepted: 29 October 2021 / Published online: 10 November 2021

(c) The Author(s) 2021

\begin{abstract}
An impaired immune response could play a role in the acquisition of secondary infections in critically ill children. Human leukocyte antigen-DR expression on monocytes (mHLA-DR) has been proposed as marker to detect immunosuppression, but its potential to predict secondary infections in critically ill children is unclear. We aimed to assess the association between mHLA-DR expression at several timepoints and the change of mHLA-DR expression over time with the acquisition of secondary infections in critically ill children. In this prospective observational study, children $<18$ years with fever and/or suspected infection (community-acquired or hospital-acquired) were included at a paediatric intensive care unit in the Netherlands. mHLA-DR expression was determined by flow cytometry on day 1, day 2-3 and day 4-7. The association between delta-mHLA-DR expression (difference between last and first measurement) and secondary infection was assessed by multivariable regression analysis, adjusted for age and Paediatric Logistic Organ Dysfunction-2 score. We included 104 patients at the PICU (median age 1.2 years [IQR 0.3-4.2]), of whom 28 patients (27\%) developed a secondary infection. Compared to 93 healthy controls, mHLA-DR expression of critically ill children was significantly lower at all timepoints. mHLA-DR expression did not differ at any of the time points between patients with and without secondary infection. In addition, delta-mHLA-DR expression was not associated with secondary infection (aOR 1.00 [95\% CI 0.96-1.04]).

Conclusions: Our results confirm that infectious critically ill children have significantly lower mHLA-DR expression than controls. mHLA-DR expression was not associated with the acquisition of secondary infections.

\section{What is Known:}

- An impaired immune response, estimated by mHLA-DR expression, could play an essential role in the acquisition of secondary infections in critically ill children.

- In critically ill children, large studies on the association of mHLA-DR expression with secondary infections are scarce.

What is New:

- Our study confirms that critically ill children have lower mHLA-DR expression than healthy controls.

- $m H L A-D R$ expression and change in mHLA-DR was not associated with the acquisition of secondary infection.
\end{abstract}

Keywords Immunosuppression $\cdot$ Secondary infection $\cdot$ Critical care $\cdot$ Pediatrics

\author{
Abbreviations \\ GM-CSF Granulocyte-macrophagecolony-stimulation \\ factor \\ mHLA-DR Humanleukocyte antigen-DR expression on \\ monocyt \\ Communicated by Nicole Ritz \\ Clementien Vermont \\ c.vermont@erasmusmc.nl \\ Extended author information available on the last page of the article
}

\author{
PERFORM Personalised Riskassessment in Febrile \\ illness to optimise Real-Life Management \\ across theEuropean Union \\ PICU Paediatric intensivecare unit \\ PRISM Paediatric risk ofMortality \\ PELOD-2 Paediatric LogisticOrgan Dysfunction-2
}




\section{Introduction}

Critically ill patients are at risk for a prolonged period of immunosuppression, potentially leading to an increased risk of secondary infections [1-4]. Secondary infections, acquisition of an infection during hospital admission, occur in 11 to $19 \%$ of paediatric intensive care unit (PICU) admissions [5-7], causing prolonged hospital stay, morbidity, mortality and costs [8].

Immunosuppression has been established in critically ill patients following trauma, surgery or stroke [9-14], but most studies have focused on adult patients with sepsis and septic shock [3, 15-22].

Monocytic human leukocyte antigen-DR (mHLA-DR) expression has been shown a reliable biomarker in critically ill patients to estimate immunosuppression. In adults, a prolonged decrease in mHLA-DR expression has been associated with acquisition of secondary infection and mortality in small studies [13, 19, 20, 23, 24] although one study did not found an association [25]. The largest study to date included $>400$ adult patients and found lower mHLA-DR values in non-survivors. However, the authors concluded that mHLA-DR expression was not suitable as predictive parameter due to limited discriminative ability [26].

In children, small studies have confirmed an association of reduced mHLA-DR with secondary infection or mortality in post-operative critically ill children [27], in children with critical illness for multiple reasons [28] and in septic children $[16,18]$. In 30 paediatric septic patients, Manzoli et al. found an association of decreased mHLA-DR expression with mortality but not with secondary infections [18]. On our PICU, critically ill children are admitted with a wide range of infectious diseases including suspected community-acquired infections and hospital-acquired infections. More insight in mHLA-DR expression and the relation with mortality and secondary infections in these children can aid to identify patients who could benefit from immunostimulatory therapies.

Therefore, we performed a large study including children with a wide range of infectious diseases to assess the relation of mHLA-DR expression with adverse outcomes. The association of mHLA-DR expression at several timepoints and the change of mHLA-DR expression over time was studied and related to mortality and the acquisition of secondary infections.

\section{Methods}

\section{Study design and population}

This is a pre-planned prospective observational study embedded in the PERFORM project (Personalised Risk assessment in
Febrile illness to optimise Real-Life Management across the European Union, www. perform2020.org) [29]. The overarching aim of PERFORM is to improve diagnosis and management of febrile children by development of a new diagnostic tests to discriminate bacterial from viral infections. For this particular study, we included critically ill children (aged 0-17 years) admitted to the level 3 PICU of Erasmus MC-Sophia Children's hospital (Rotterdam, the Netherlands, mixed surgical/medical) between March 2017 and April 2019. Inclusion criteria included fever (body temperature of $\geq 38^{\circ} \mathrm{C}$ ) and/or a suspected infection in patients who had an arterial or central venous line in situ. This comprised both children who were admitted through the Emergency Department (community-acquired infection) and children who were already admitted and developed an infection during hospital admission $>48 \mathrm{~h}$ after admission (hospitalacquired infection). For critically ill children, exclusion criteria were presence of chronic conditions that affect immune status (immunodeficiency, malignancy, chronic immunosuppressive medication including corticosteroids) and severe anaemia (haemoglobin $<6.5 \mathrm{~g} / \mathrm{dL}$ ). In addition, we included afebrile healthy controls who underwent elective surgery for a minor condition. For healthy controls, exclusion criteria included fever in the three week prior to surgery or presence of more than one chronic condition. To cover all ages, we aimed to include 20 healthy children in clinically relevant age groups: $0-1$ years, $1-2$ years, $2-5$ years, 5-12 years and 12-16 years. Informed consent was obtained from children $>12$ years and parents or legal guardians.

\section{Clinical data}

Prospective clinical data were collected from electronic medical records. We collected baseline data on the first day of PICU admission or, in a case of suspected hospital-acquired infection, at the onset of the infectious disease episode on the PICU. Collected data included demographics, chronic conditions, Paediatric Risk of Mortality (PRISM) score [30], Paediatric Logistic Organ Dysfunction-2 (PELOD-2) probability of mortality [31] and signs of septic shock (sepsis and cardiovascular organ dysfunction as defined by Goldstein et al.) [32]. The cause of the initial infection was classified in proven/presumed bacterial, proven/presumed viral, unknown bacterial/viral or other, according to a published flowchart including clinical signs and symptoms and microbiological cultures/PCR [33]. In addition, for a period of 28 days following inclusion, we collected days of mechanical ventilation, days of inotropic support, survival status and number of PICU-free days at day 28.

\section{Outcome measures}

The outcome measures included 28-day mortality and acquisition of a secondary infection defined by the 
surveillance definition of hospital-acquired infections by Centres for Disease Control and Prevention according to previous studies [7, 28, 34]. All suspected secondary infections during 28 days of follow-up after inclusion were reviewed by trained clinical researchers $(\mathrm{NH}, \mathrm{PK}, \mathrm{JW})$ and discussed with one paediatric infectious disease specialist (CV). All researchers were blinded regarding mHLA-DR expression at the time of reviewing.

\section{Blood sampling and measurement of monocytic HLA-DR expression}

Blood samples were taken either on PICU admission or at the onset of the infectious episode during PICU stay. Follow-up samples were taken at day 2-3, day 4-7 and once a week with a maximum of 5 blood draws in total. The number of time points in which follow-up sampling could be performed was restricted by logistic reasons, limitations of blood sample volumes and lack of parents' consent. For healthy controls, a single sample was drawn during insertion of the peripheral catheter before start of surgery. Samples were analysed for mHLA-DR expression on a flowcytometer (FACSCanto-II, BectonDickinson) using the Anti-HLA-DR/ Anti-Monocyte Quantibriteassay (BD Biosciences), as described previously $[35,36]$. This assay approach uses an HLA-DR antibody conjugated to phycoerythrin (PE) in a 1:1 ratio as well as a mixture of beads to which a defined amount of PE molecules have been conjugated. In the end, this allows to calculate the number of HLA-DR-PE antibodies bound per cell $(\mathrm{AB} / \mathrm{c})$. Laboratory technicians conducting the assay were blinded for the clinical data.

\section{Data analysis}

We compared results of mHLA-DR expression of patients with suspected infections vs healthy controls, and patients with and without secondary infections. Differences between the groups were tested for significance with Mann-Whitney $U$ test, Student's $t$ test or Kruskal-Wallis test when appropriate. To assess the change of mHLA-DR expression over time during PICU admission, we calculated delta-mHLA-DR: the difference of mHLA-DR expression between the latest timepoint and the first timepoint.

In logistic regression analysis, we adjusted for age and baseline PELOD-2 score. We assessed the association of delta-mHLA-DR for acquisition of secondary infections, and for the composite outcome acquisition of secondary infections and/or 28-day mortality. Based on delta-mHLADR, patients were classified as improved (delta $>20 \%$ from baseline), declined (delta $<-20 \%$ from baseline) or stable $(<20 \%$ difference from baseline). The delta $20 \%$ from baseline was chosen to ensure sufficient numbers for statistical analysis. We tested the interaction between delta-mHLA-DR and delta-mHLA-DR group (improved, declined, stable) for secondary infection using the likelihood ratio test. In addition, we tested the interaction between delta-mHLA-DR and cause of the initial infection (proven/presumed bacterial, proven/presumed viral, unknown bacterial/viral or other). Next, we explored the association of delta-mHLA-DR in the subgroup with low mHLA-DR, defined as values below the $25^{\text {th }}$ centile at baseline in our population. As an exploratory analysis, we assessed the association of delta-mHLA-DR with PICU free days at day 28 adjusted for age and baseline PELOD-2 score. All data analyses were performed in $\mathrm{R}$ version 3.6 and a $p$ value $<0.05$ was considered significant.

\section{Results}

\section{Study population}

We included 104 patients with suspected infection and 93 healthy controls with available mHLA-DR measurements. Compared to controls, infectious patients were younger (median 1.2 years [IQR 0.3-4.2] vs 3.6 years [IQR 1.1-9.9]) but were similar in sex ((male: 60\% (62/104) vs 66\% (61/93)) (additional file 1). Of the 104 infectious patients, the initial infection was a suspected community-acquired infection in $36 \%(n=37)$ and a suspected hospital-acquired infection in $64 \%(n=76)$. Details of the clinical syndromes are presented in additional file 2 . Seven patients died (7\%) and 28 patients (27\%) acquired a secondary infection after inclusion which occurred after a median of 9 days (IQR 5-16 days) (Fig. 1, additional file 3). Compared to patients without a secondary infection, patients with a secondary infection were similar in age, sex, initial infection and baseline PELOD-2 and PRISM score. Patients with a secondary infection, however, had more ventilation and inotropes days and were admitted longer to the PICU (Table 1) than patients without a secondary infection. Comparing children with suspected community-acquired and hospital-acquired infections, no differences on baseline PRISM or PELOD-2 scores or occurrence of secondary infections were observed.

\section{Monocytic HLA-DR expression}

In controls, mHLA-DR expression was 29,500 AB/cell (IQR 23,800-37,600) and was similar across age groups (0-1 years 28,000 [IQR 26,100-39300]; 1-2 years 34,300 [25700-42600]; 2-5 years 33,900 [27600-43800]; 5-12 years 28,000 [24900-35800]; $12-18$ years 23,600 [19700-28300]) (Fig. 2a). In controls, boys had higher mHLA-DR expression than girls $(32,900 \mathrm{AB} /$ cell [IQR $25,700-43300$ ] vs 25,400 [IQR 21,400-32200], $p<0.01$ ) (Fig. 2b), but mHLA-DR expression in critically ill 
Fig. 1 Cumulative incidence of secondary infection $(n=104)$

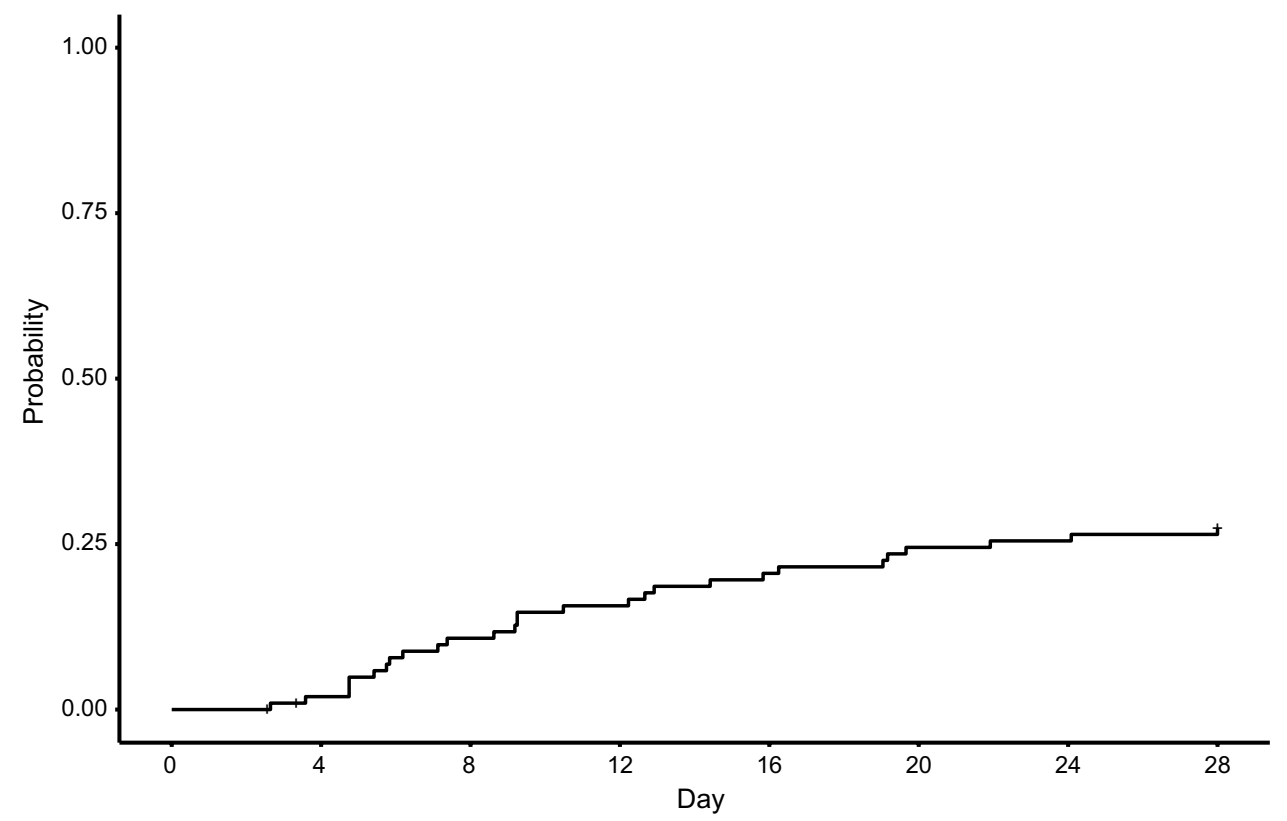

Table 1 Descriptive characteristics of critically ill children with suspected infections $(n=104)$

\begin{tabular}{|c|c|c|c|}
\hline & $\begin{array}{l}\text { Secondary infection } \\
n=28\end{array}$ & $\begin{array}{l}\text { No secondary infection } \\
n=76\end{array}$ & $p$ value \\
\hline Age in years, median [IQR] & $1.3(0.5-3.1)$ & $1.0(0.2-4.2)$ & 0.47 \\
\hline Male & $18(64.3)$ & $44(57.9)$ & 0.72 \\
\hline Any chronic underlying condition* & $21(75.0)$ & $54(71.1)$ & 0.88 \\
\hline Pulmonary & $5(17.9)$ & $11(14.5)$ & \\
\hline Prematurity & $2(7.1)$ & $7(9.2)$ & \\
\hline Gastro-intestinal & & $3(3.9)$ & \\
\hline Neurological & $2(7.1)$ & $7(9.2)$ & \\
\hline Cardio & $15(53.6)$ & $36(47.4)$ & \\
\hline Endocrinologic & $1(3.6)$ & & \\
\hline Genetic & $6(21.4)$ & $9(11.8)$ & \\
\hline PRISM score at day 1, median [IQR] & $15(12-22)$ & $14(9-19)$ & 0.26 \\
\hline PELOD-2 probability of mortality at day 1 , median [IQR] & $2.2(1.3-6.2)$ & $1.4(0.5-3.5)$ & 0.11 \\
\hline PICU admission duration in days during study period, median [IQR] & $19(9-36)$ & $8(2-17)$ & 0.003 \\
\hline Days of invasive ventilation during study period & $7(2-18)$ & $2(0-5)$ & 0.003 \\
\hline Days of inotropes during study period & $2(0-9)$ & $1(0-2)$ & 0.02 \\
\hline Signs of septic shock at baseline & $9(32.1)$ & $15(19.7)$ & 0.26 \\
\hline Phenotype initial infection & & & 0.08 \\
\hline Proven/presumed bacterial infections $\mathrm{s}^{\mathrm{a}}$ & $13(46.4)$ & $19(25.0)$ & \\
\hline Proven/presumed viral infections ${ }^{\mathrm{b}}$ & $3(10.7)$ & $16(21.1)$ & \\
\hline Unknown bacterial/viral & $3(10.7)$ & $6(7.9)$ & \\
\hline Other & $9(32.1)$ & $35(46.1)$ & \\
\hline Mortality & $4(14.3)$ & $2(2.6)$ & 0.07 \\
\hline
\end{tabular}

$I Q R$, interquartile range; PELOD-2, Paediatric Logistic Organ Dysfunction-2; PRISM, Paediatric Risk of Mortality

*Multiple categories possible for one patient

${ }^{\mathrm{a}} n=18$ proven bacterial infections ( $n=5$ for secondary infection, $n=13$ for no secondary infection)

${ }^{\mathrm{b}} n=2$ proven viral infections ( $n=0$ for secondary infection, $n=2$ for no secondary infection) 
children was similar in girls and boys at all timepoints. In critically ill children, mHLA-DR expression was significantly lower than in controls at all timepoints (Fig. 2c). No differences were observed for baseline mHLA-DR expression in community-acquired infections or hospitalacquired infections.

\section{Association of monocytic HLA-DR expression with secondary infection}

mHLA-DR expression did not differ for patients with and without secondary infections at any of the timepoints (Fig. 3a). In patients with two or more measurements $(n=47)$, delta-mHLA-DR varied widely (range $-45,232$ to $40,944 \mathrm{AB} /$ cell). Delta-mHLA-DR expression did not differ for patients with and without secondary infection (712,000 AB/cell [IQR - 496 to 4371] vs $(497,000 \mathrm{AB} /$ cell [IQR - 5161 to 4896]) (Fig. 3b). Adjusted for age and PELOD-2 score, delta-mHLA-DR was not associated with acquisition of secondary infection (aOR 1.00 [95\% CI 0.96-1.04]), or the composite outcome secondary infection/mortality (aOR 0.99 [0.96-1.04]). Occurrence of secondary infection did not differ for patients classified by delta-mHLA-DR as improved $(8 / 19,42 \%)$, declined $(4 / 14,29 \%)$ or stable $(7 / 14,50 \%)$, although numbers were small (Fig. 3c) ( $p>0.05$ for interaction). In addition, the association of delta-mHLA-DR did not vary for cause of infection groups ( $p>0.05$ for interaction). In the subgroup of patients with low mHLA-DR at baseline $(<7200 \mathrm{AB} /$ cell, $n=26$ ), delta-mHLA-DR was not associated with secondary infection (OR 1.0 [95\% CI 0.7-1.6]). Lastly, adjusted for age and PELOD-2 score, delta-mHLA-DR was not associated with PICU free days at day 28 ( $\beta 0.05[95 \%$ CI -0.15 to 0.26$])$.
A

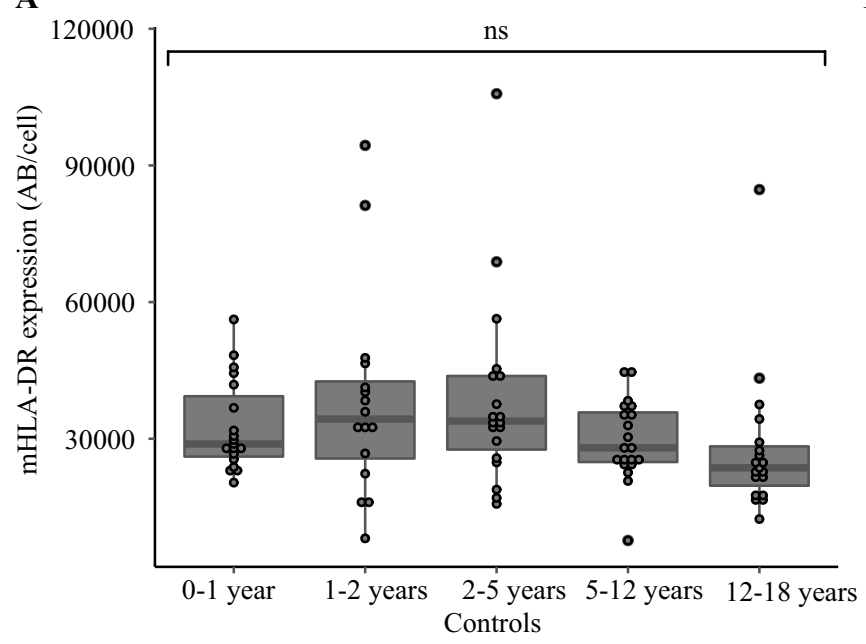

B

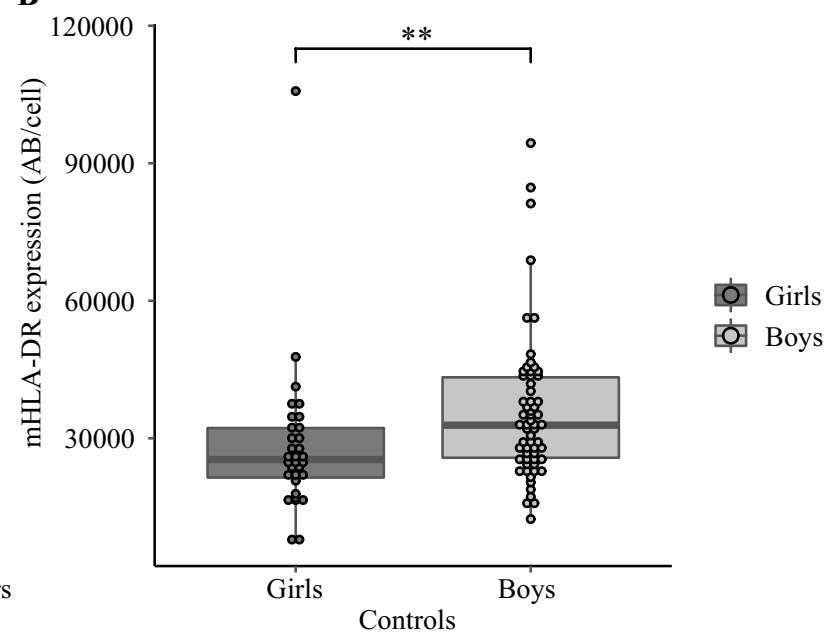

C

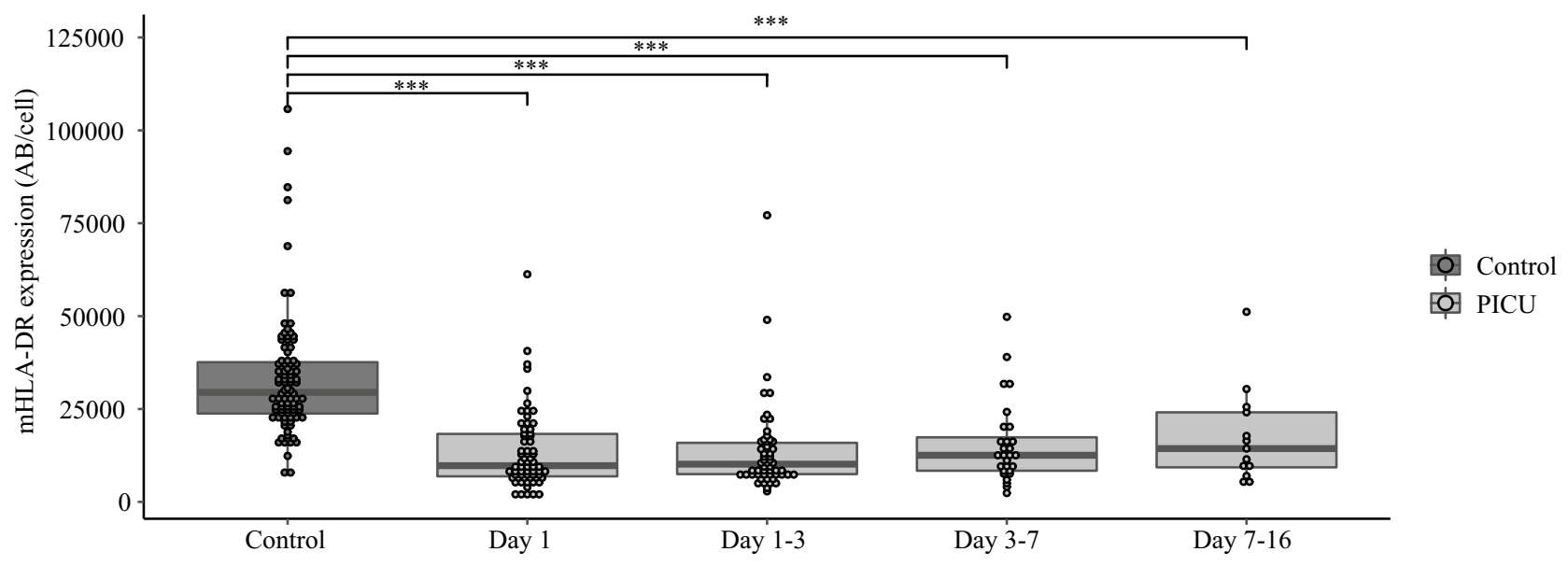

Fig. 2 Monocyte HLA-DR expression. A mHLA-DR expression stratified for age group in controls. B mHLA-DR expression stratified for sex in controls. C mHLA-DR expression for controls and children with suspected infections at different timepoints. Ns, not significant; $* * * p<0.001$, ** $p<0.01$ 


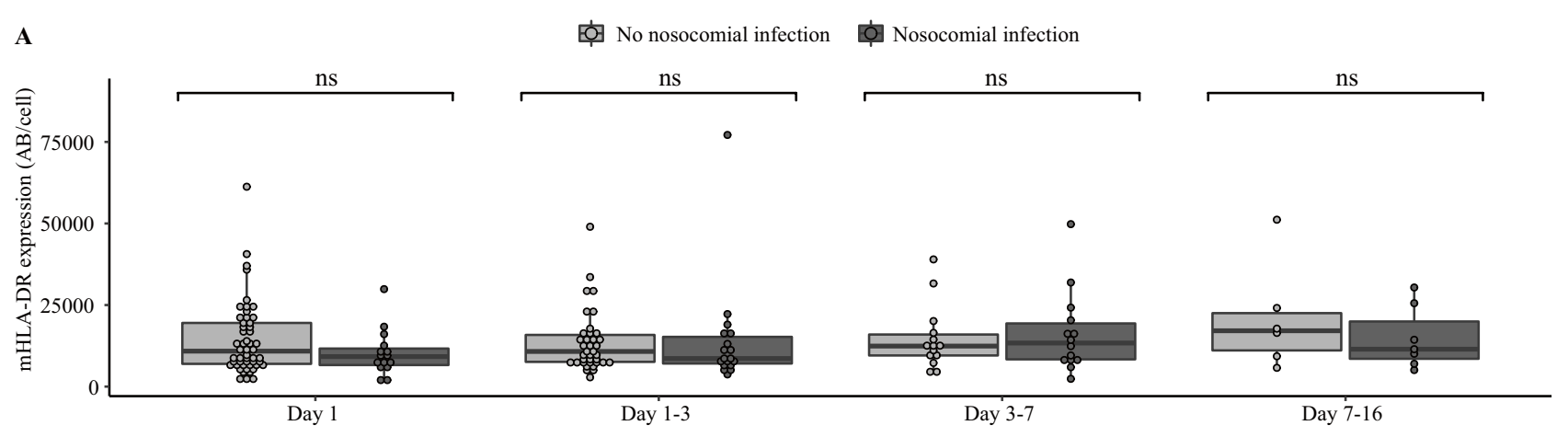

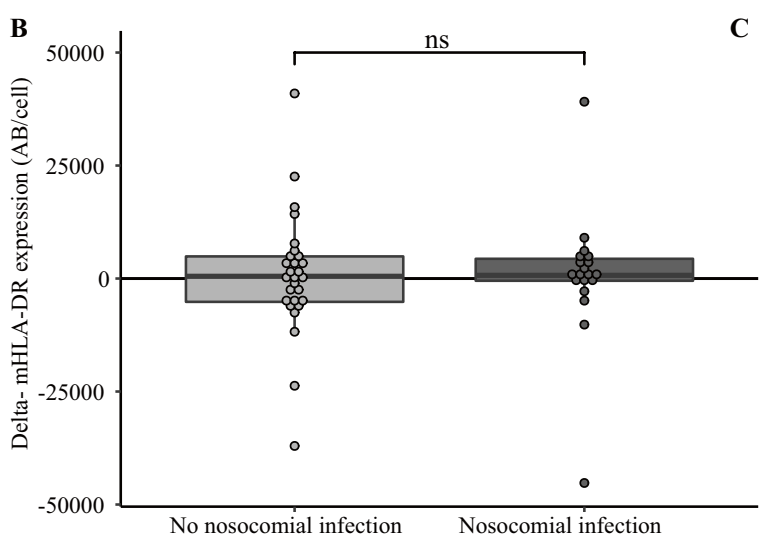

Fig. 3 Secondary infection. A mHLA-DR expression stratified for secondary infections at different timepoints. B delta-mHLADR expression in patients with and without secondary infec-

\section{Discussion}

\section{Main findings}

In our study of critically ill children with suspected infections, mHLA-DR expression was significantly lower compared to healthy controls. No association was found between mHLA-DR expression and acquisition of secondary infections at any of the timepoints. In addition, change in mHLADR expression over time was not associated with the occurrence of secondary infections or the composite outcome secondary infection/mortality.

Our study also provides reference values of mHLA-DR expression in healthy children in different age groups and, similar to previous studies, we found no difference of mHLA-DR expression for age. Compared to one previous study in children using the same measurement method, we found similar mHLA-DR values in our large sample of controls [18]. Surprisingly, healthy boys had higher mHLA-DR expression compared to girls. A previous study including healthy children did not report any differences in sex for mHLA-DR expression [28]. This sex-difference in mHLA-DR was not observed in critically ill children, although their mHLA-DR levels were

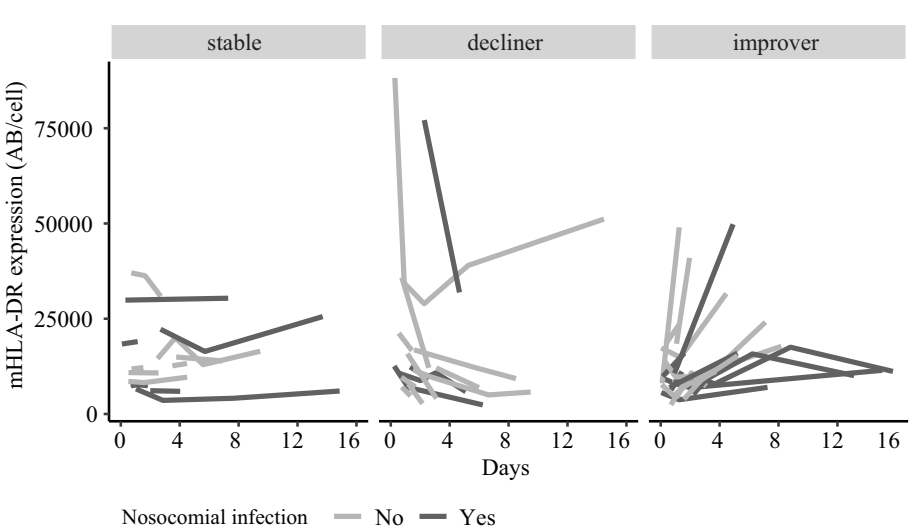

tion. $\mathbf{C}$ mHLA-DR expression in secondary infection stratified for delta-mHLA-DR groups (stable, decliner, improver). Ns, not significant

also influenced by disease severity. In two studies investigating immune markers in adults, pre-surgical mHLA-DR levels did not differ between men and women $[37,38]$. As mHLA-DR expression did not differ in the various studied age groups, we did not perform an age-matched analysis.

Previous studies in children have investigated the relation of mHLA-DR expression with secondary infections in general ICU admissions or in septic shock: Boeddha et al. performed in our hospital, and Remy et al. reported a significant association, whereas Manzoli et al. did not [16, 18, 28]. Our study, using a larger cohort of children, did not show an association of mHLA-DR expression with secondary infection. Populations included in previous studies differed: they were based on small number of patients (range 30-37 patients) and included either solely septic patients or a general ICU population, whereas our study included children with suspected infections. We included both (suspected) community-acquired and hospital-acquired infections, to cover the wide range of suspected infections at the ICU. Although these two groups might have different immunological profiles or different risks for secondary infections, we did not observe differences in baseline mHLA-DR expression or occurrence of secondary infections. In our study, 
the proportion of patients who suffered from a secondary infection (28\%) was higher than reported by other studies including general PICU populations (11-19\%) [5-7].

Although it is widely established that mHLA-DR expression is a measure for immunosuppression, cut-off values for mHLA-DR defining immunosuppression in both adults and children are yet unclear. In different adult trials which investigated immunostimulatory therapies in septic patients, cutoffs of $<8000 \mathrm{mAB} /$ cell and 10,000 AB/cell were used for patient selection [40; 41]. Tamulyte et al. found that different cut-off values for mHLA-DR expression (2000/5000/8000 molecules/cell) could not predict mortality, but the cut-offs of 2000 and 5000 could discriminate patients with a longer ICU stay and ventilation days. In their study, mHLA-DR measurement was performed using novel point-of-care flow cytometry. It is yet unclear whether these cut-off values can be extrapolated to children. Manzoli et al. suggested that a delta of $<1000 \mathrm{AB} /$ cell was associated with mortality in septic children [18]. We hypothesized that different cut-offs may be needed for prediction of secondary infections. Therefore, we performed a subgroup analysis in the lowest mHLA-DR quartile but found no association of change in mHLA-DR expression with the occurrence of secondary infection.

Although we found that mHLA-DR expression is lower in our cohort of critically ill children compared to healthy controls, its clinical value is yet unclear. Our results show that in critically ill children with suspected infections, mHLA-DR expression has no additional value to identify patients who are at risk for secondary infection. The immunosuppressed state of critically ill children as measured by mHLA-DR was not related to acquisition of secondary infections or with duration of ICU stay.

Previous studies have used immunostimulating therapies as granulocyte-macrophage colony-stimulation factor (GM-CSF) to restore mHLA-DR levels and improved patient outcomes [39-43]. In addition, interferon-gamma improved the immune response in a child suffering from refractory candidemia with extremely low mHLA-DR [44]. Currently, these immunostimulating therapies are not routinely used in clinical practice due to the lack of predictive ability on the individual level $[26,45]$. Hence, future trials in children should focus on accurate identification of immunosuppression using point-of-care devices in the assessment of mHLA-DR and prediction could be improved by using multiple biomarkers involved in other pathways such as programmed death (PD)-1 [46].

The main strengths of this study include its prospective design and inclusion of large number of critically ill children with a wide variety of infections. Second, we collected detailed clinical data with complete follow-up on all patients. In addition, we used a standardized method for mHLA-DR measurement to facilitate comparison with other studies $[35,36]$. The major limitation of this study is that we were not able to collect follow-up samples in all patients: patients were either discharged from the PICU, had their central line removed, no consent was given for multiple blood sampling, blood volume restrictions were reached or mHLA-DR measurement was not possible due to logistic reasons. It is possible that follow-up samples were collected in critically ill children with more severe disease, which could limit the generalizability of our results to the general PICU population. Furthermore, secondary infections may occur more frequent in patients who have more ventilation days, more ICU days or invasive medical devices such as urinary catheters and central venous catheters. Although we adjusted for PELOD-2 in our analysis, we did not have enough power to include all these confounders in our analysis. Lastly, some patients in our cohort had surgery during the follow-up period which could have influenced mHLA-DR levels [27].

\section{Conclusion}

In this single-centre study of mHLA-DR expression in children admitted to PICU with suspected infections, we confirm that critically ill children have lower mHLADR expression than controls. Decreased mHLA-DR and change in mHLA-DR was not associated with the acquisition of secondary infections. Therefore, in this population, mHLA-DR expression is not valuable for identifying children at risk for secondary infections.

Supplementary information The online version contains supplementary material available at https://doi.org/10.1007/s00431-021-04313-7.

Acknowledgements We thank Josephine Wagenaar (former medical student) for her help in data quality assessment.

Authors' contributions Conceptualization: NNH, GD, WAD, CV, Data curation: NNH, PK, NMAN, SV, KFJ, HM, GD, WAD, CV, Data analysis: NNH, DN, CV, Supervision: CV, Writing - original draft: NNH, Writing - review \& editing: PK, NMAN, DN, SV, KFJ, HM, GD, WAD, CV

Funding The PERFORM study has received funding from the European Union's Horizon 2020 research and innovation programme under grant agreement No. 668303

Availability of data and materials A data set containing individual participant data will be made available in a public data repository containing a specific DOI upon publication. The data will be anonymized and will not contain any identifiable data.

\section{Declarations}

Ethics approval The PERFORM study protocol was approved by the local Medical Ethics Review Board (MERB) (CMO ArnhemNijmegen, NL58103.091.16, 2016-3085). 
Consent to participate Written informed consent was obtained for all participants.

Consent to publication Not applicable.

Competing interests The authors have no relevant financial or nonfinancial interests to disclose.

Open Access This article is licensed under a Creative Commons Attribution 4.0 International License, which permits use, sharing, adaptation, distribution and reproduction in any medium or format, as long as you give appropriate credit to the original author(s) and the source, provide a link to the Creative Commons licence, and indicate if changes were made. The images or other third party material in this article are included in the article's Creative Commons licence, unless indicated otherwise in a credit line to the material. If material is not included in the article's Creative Commons licence and your intended use is not permitted by statutory regulation or exceeds the permitted use, you will need to obtain permission directly from the copyright holder. To view a copy of this licence, visit http://creativecommons.org/licenses/by/4.0/.

\section{References}

1. Hall MW, Geyer SM, Guo CY, Panoskaltsis-Mortari A, Jouvet P, Ferdinands J, Shay DK, Nateri J, Greathouse K, Sullivan R, Tran T, Keisling S, Randolph AG, Pediatric Acute Lung I, Sepsis Investigators Network PSI (2013) Innate immune function and mortality in critically ill children with influenza: a multicenter study. Crit Care Med 41:224-236

2. Leentjens J, Kox M, van der Hoeven JG, Netea MG, Pickkers P (2013) Immunotherapy for the adjunctive treatment of sepsis: from immunosuppression to immunostimulation. Time for a paradigm change?. Am J Respir Crit Care Med 187:1287-1293

3. Hotchkiss RS, Monneret G, Payen D (2013) Immunosuppression in sepsis: a novel understanding of the disorder and a new therapeutic approach. Lancet Infect Dis 13:260-268

4. Otto GP, Sossdorf M, Claus RA, Rödel J, Menge K, Reinhart $\mathrm{K}$, Bauer M, Riedemann NC (2011) The late phase of sepsis is characterized by an increased microbiological burden and death rate. Crit Care 15:R183

5. Asembergiene J, Gurskis V, Kevalas R, Valinteliene R (2009) Nosocomial infections in the pediatric intensive care units in Lithuania. Medicina (Kaunas) 45:29-36

6. Chomton M, Brossier D, Sauthier M, Vallières E, Dubois J, Emeriaud G, Jouvet P (2018) Ventilator-associated pneumonia and events in pediatric intensive care: a single center study. Pediatr Crit Care Med 19:1106-1113

7. Fivez T, Kerklaan D, Mesotten D, Verbruggen S, Wouters PJ, Vanhorebeek I, Debaveye Y, Vlasselaers D, Desmet L, Casaer MP, Garcia Guerra G, Hanot J, Joffe A, Tibboel D, Joosten K, Van den Berghe G (2016) Early versus late parenteral nutrition in critically ill children. N Engl J Med 374:1111-1122

8. van Puffelen E, Polinder S, Vanhorebeek I, Wouters PJ, Bossche N, Peers G, Verstraete S, Joosten KFM, Van den Berghe G, Verbruggen S, Mesotten D (2018) Cost-effectiveness study of early versus late parenteral nutrition in critically ill children (PEPaNIC): preplanned secondary analysis of a multicentre randomised controlled trial. Crit Care 22:4

9. Flohé S, Lendemans S, Schade FU, Kreuzfelder E, Waydhas C (2004) Influence of surgical intervention in the immune response of severely injured patients. Intensive Care Med 30:96-102
10. Tamulyte S, Kopplin J, Brenner T, Weigand MA, Uhle F (2019) Monocyte HLA-DR assessment by a novel point-of-care device is feasible for early identification of ICU patients with complicated courses-a proof-of-principle study. Front Immunol 10:432

11. Muszynski JA, Nofziger R, Greathouse K, Nateri J, Hanson-Huber L, Steele L, Nicol K, Groner JI, Besner GE, Raffel C, Geyer S, El-Assal O, Hall MW (2014) Innate immune function predicts the development of nosocomial infection in critically injured children. Shock 42:313-321

12. Gessler P, Pretre R, Bürki C, Rousson V, Frey B, Nadal D (2005) Monocyte function-associated antigen expression during and after pediatric cardiac surgery. J Thorac Cardiovasc Surg 130:54-60

13. Venet F, Tissot S, Debard AL, Faudot C, Crampé C, Pachot A, Ayala A, Monneret G (2007) Decreased monocyte human leukocyte antigen-DR expression after severe burn injury: correlation with severity and secondary septic shock. Crit Care Med 35:1910-1917

14. Zhang DP, Yan FL, Xu HQ, Zhu YX, Yin Y, Lu HQ (2009) A decrease of human leucocyte antigen-DR expression on monocytes in peripheral blood predicts stroke-associated infection in critically-ill patients with acute stroke. Eur J Neurol 16:498-505

15. Hotchkiss RS, Monneret G, Payen D (2013) Sepsis-induced immunosuppression: from cellular dysfunctions to immunotherapy. Nat Rev Immunol 13:862-874

16. Remy S, Kolev-Descamps K, Gossez M, Venet F, Demaret J, Javouhey E, Monneret G (2018) Occurrence of marked sepsisinduced immunosuppression in pediatric septic shock: a pilot study. Ann Intensive Care 8:36

17. Leijte GP, Rimmelé T, Kox M, Bruse N, Monard C, Gossez M, Monneret G, Pickkers P, Venet F (2020) Monocytic HLA-DR expression kinetics in septic shock patients with different pathogens, sites of infection and adverse outcomes. Crit Care 24:110

18. Manzoli TF, Troster EJ, Ferranti JF, Sales MM (2016) Prolonged suppression of monocytic human leukocyte antigen-DR expression correlates with mortality in pediatric septic patients in a pediatric tertiary Intensive Care Unit. J Crit Care 33:84-89

19. Wu JF, Ma J, Chen J, Ou-Yang B, Chen MY, Li LF, Liu YJ, Lin AH, Guan XD (2011) Changes of monocyte human leukocyte antigen-DR expression as a reliable predictor of mortality in severe sepsis. Crit Care 15:R220

20. Monneret G, Lepape A, Voirin N, Bohé J, Venet F, Debard AL, Thizy H, Bienvenu J, Gueyffier F, Vanhems P (2006) Persisting low monocyte human leukocyte antigen-DR expression predicts mortality in septic shock. Intensive Care Med 32:1175-1183

21. Drewry AM, Ablordeppey EA, Murray ET, Dalton CM, Fuller BM, Kollef MH, Hotchkiss RS (2018) Monocyte function and clinical outcomes in febrile and afebrile patients with severe sepsis. Shock 50:381-387

22. Venet F, Lukaszewicz A-C, Payen D, Hotchkiss R, Monneret $\mathrm{G}$ (2013) Monitoring the immune response in sepsis: a rational approach to administration of immunoadjuvant therapies. Curr Opin Immunol 25:477-483

23. Drewry AM, Ablordeppey EA, Murray ET, Beiter ER, Walton AH, Hall MW, Hotchkiss RS (2016) Comparison of monocyte human leukocyte antigen-DR expression and stimulated tumor necrosis factor alpha production as outcome predictors in severe sepsis: a prospective observational study. Crit Care 20:334

24. Tschaikowsky K, Hedwig-Geissing M, Schiele A, Bremer F, Schywalsky M, Schüttler J (2002) Coincidence of pro- and anti-inflammatory responses in the early phase of severe sepsis: longitudinal study of mononuclear histocompatibility leukocyte antigen-DR expression, procalcitonin, $\mathrm{C}$-reactive protein, and changes in T-cell subsets in septic and postoperative patients. Crit Care Med 30:1015-1023 
25. Perry SE, Mostafa SM, Wenstone R, Shenkin A, McLaughlin PJ (2003) Is low monocyte HLA-DR expression helpful to predict outcome in severe sepsis?. Intensive Care Med 29:1245-1252

26. Trimmel H, Luschin U, Köhrer K, Anzur C, Vevera D, Spittler A (2012) Clinical outcome of critically ill patients cannot be defined by cutoff values of monocyte human leukocyte antigenDR expression. Shock 37:140-144

27. Allen ML, Peters MJ, Goldman A, Elliott M, James I, Callard R, Klein NJ (2002) Early postoperative monocyte deactivation predicts systemic inflammation and prolonged stay in pediatric cardiac intensive care. Crit Care Med 30:1140-1145

28. Boeddha NP, Kerklaan D, Dunbar A, van Puffelen E, Nagtzaam NMA, Vanhorebeek I, Van den Berghe G, Hazelzet JA, Joosten KF, Verbruggen SC, Dik WA, Driessen GJ (2018) HLA-DR expression on monocyte subsets in critically ill children. Pediatr Infect Dis J 37:1034-1040

29. PERFORM consortium (2019) Personalised risk assessment in febrile illness to optimise real-life management (PERFORM). PERFORM

30. Pollack MM, Ruttimann UE, Getson PR (1988) Pediatric risk of mortality (PRISM) score. Read Online: Critic Care Medic I Soc Critic Care Medic 16

31. Leteurtre S, Duhamel A, Salleron J, Grandbastien B, Lacroix J, Leclerc F, Groupe Francophone de Reanimation et d'Urgences P (2013) PELOD-2: an update of the PEdiatric logistic organ dysfunction score. Crit Care Med 41:1761-1773

32. Goldstein B, Giroir B, Randolph A (2005) International Consensus Conference on Pediatric S International pediatric sepsis consensus conference: definitions for sepsis and organ dysfunction in pediatrics. Pediatr Crit Care Med 6:2-8

33. Hagedoorn NN, Borensztajn DM, Nijman R, Balode A, von Both U, Carrol ED, Eleftheriou I et al (2020) Variation in antibiotic prescription rates in febrile children presenting to emergency departments across Europe (MOFICHE): a multicentre observational study. PLoS Medic 17:e1003208

34. Horan TC, Andrus M, Dudeck MA (2008) CDC/NHSN surveillance definition of health care-associated infection and criteria for specific types of infections in the acute care setting. Am J Infect Control 36:309-332

35. Demaret J, Walencik A, Jacob MC, Timsit JF, Venet F, Lepape A, Monneret G (2013) Inter-laboratory assessment of flow cytometric monocyte HLA-DR expression in clinical samples. Cytometry B Clin Cytom 84:59-62

36. Döcke WD, Höflich C, Davis KA, Röttgers K, Meisel C, Kiefer P, Weber SU, Hedwig-Geissing M, Kreuzfelder E, Tschentscher P, Nebe T, Engel A, Monneret G, Spittler A, Schmolke K, Reinke P, Volk HD, Kunz D (2005) Monitoring temporary immunodepression by flow cytometric measurement of monocytic HLADR expression: a multicenter standardized study. Clin Chem $51: 2341-2347$
37. Lachmann G, von Haefen C, Kurth J, Yuerek F, Wernecke KD, Spies C (2017) Smoking, gender, and overweight are important influencing factors on monocytic HLA-DR before and after major cancer surgery. Biomed Res Int 2017:5216562

38. Ono S, Tsujimoto H, Hiraki S, Takahata R, Kinoshita M, Mochizuki H (2005) Sex differences in cytokine production and surface antigen expression of peripheral blood mononuclear cells after surgery. Am J Surg 190:439-444

39. Meisel C, Schefold JC, Pschowski R, Baumann T, Hetzger K, Gregor J, Weber-Carstens S, Hasper D, Keh D, Zuckermann H, Reinke P, Volk HD (2009) Granulocyte-macrophage colonystimulating factor to reverse sepsis-associated immunosuppression: a double-blind, randomized, placebo-controlled multicenter trial. Am J Respir Crit Care Med 180:640-648

40. Spies C, Luetz A, Lachmann G, Renius M, von Haefen C, Wernecke KD, Bahra M, Schiemann A, Paupers M, Meisel C (2015) Influence of granulocyte-macrophage colony-stimulating factor or influenza vaccination on HLA-DR, infection and delirium days in immunosuppressed surgical patients: double blind, randomised controlled trial. PLoS One 10:e0144003

41. Nierhaus A, Montag B, Timmler N, Frings DP, Gutensohn K, Jung R, Schneider CG, Pothmann W, Brassel AK, Schulte Am Esch J (2003) Reversal of immunoparalysis by recombinant human granulocyte-macrophage colony-stimulating factor in patients with severe sepsis. Intensive Care Med 29:646-651

42. Bilgin K, Yaramiş A, Haspolat K, Taş MA, Günbey S, Derman O (2001) A randomized trial of granulocyte-macrophage colonystimulating factor in neonates with sepsis and neutropenia. Pediatrics 107:36-41

43. Hall MW, Knatz NL, Vetterly C, Tomarello S, Wewers MD, Volk HD, Carcillo JA (2011) Immunoparalysis and nosocomial infection in children with multiple organ dysfunction syndrome. Intensive Care Med 37:525-532

44. Buddingh EP, Leentjens J, van der Lugt J, Dik WA, Gresnigt MS, Netea MG, Pickkers P, Driessen GJ (2015) Interferon-gamma immunotherapy in a patient with refractory disseminated candidiasis. Pediatr Infect Dis J 34:1391-1394

45. Bruse N, Leijte GP, Pickkers P, Kox M (2019) New frontiers in precision medicine for sepsis-induced immunoparalysis. Expert Rev Clin Immunol 15:251-263

46. McBride MA, Patil TK, Bohannon JK, Hernandez A, Sherwood ER, Patil NK (2021) Immune checkpoints: novel therapeutic targets to attenuate sepsis-induced immunosuppression. Front Immunol 11

Publisher's Note Springer Nature remains neutral with regard to jurisdictional claims in published maps and institutional affiliations. 


\section{Authors and Affiliations}

\section{Nienke N. Hagedoorn ${ }^{1}$. Pinar Kolukirik ${ }^{2} \cdot$ Nicole M. A. Nagtzaam $^{3} \cdot$ Daan Nieboer $^{4} \cdot$ Sascha Verbruggen $^{5}$. Koen F. Joosten ${ }^{5} \cdot$ Henriette Moll $^{1} \cdot$ Gertjan Driessen $^{6} \cdot$ Willem A. Dik $^{3} \cdot$ Clementien Vermont $^{2}$}

Nienke N. Hagedoorn

n.hagedoorn@erasmusmc.nl

Pinar Kolukirik

pinar_40@live.nl

Nicole M. A. Nagtzaam

n.nagtzaam@erasmusmc.nl

Daan Nieboer

d.nierboer@erasmusmc.nl

Sascha Verbruggen

s.verbruggen@erasmusmc.nl

Koen F. Joosten

k.joosten@erasmusmc.nl

Henriette Moll

h.a.moll@erasmusmc.nl

Gertjan Driessen

gertjan.driessen@mumc.nl
Willem A. Dik

w.dik@erasmusmc.nl

1 Department of General Pediatrics, Erasmus MC Sophia, University Medical Center Rotterdam, Rotterdam,

The Netherlands

2 Pediatric Infectious Diseases \& Immunology, Erasmus MC Sophia, University Medical Center Rotterdam, Rotterdam, The Netherlands

3 Department of Immunology, Laboratory Medical Immunology, Erasmus MC, University Medical Center Rotterdam, Rotterdam, The Netherlands

4 Department of Public Health, Erasmus University Medical Center, Rotterdam, The Netherlands

5 Pediatric Intensive Care, Erasmus MC-Sophia, University Medical Center Rotterdam, Rotterdam, The Netherlands

6 Department of Pediatrics, Maastricht University Medical Center, Maastricht, The Netherlands 